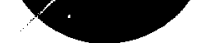

\title{
Progress Toward Standards for the Seamless Interoperability of Broadband Satellite Communication Networks
}

William D. Ivancic, Daniel R. Glover, Thomas C. vonDeak, and Kul B. Bhasin Lewis Research Center, Cleveland, Ohio

Prepared for the

17th International Communication Satellite Systems Conference cosponsored by the American Institute of Aeronautics and Astronautics and the Ministry of Posts and Telecommunications

Yokohama, Japan, February 23-27, 1998

National Aeronautics and

Space Administration

Lewis Research Center 
This report contains preliminary findings, subject to revision as analysis proceeds.

Available from

NASA Center for Aerospace Information 800 Elkridge Landing Road

Linthicum Heights, MD 21090-2934

Price Code: A03
National Technical Information Service 5287 Port Royal Road Springfield, VA 22100 Price Code: A03 


\title{
PROGRESS TOWARD STANDARDS FOR THE SEAMLESS INTEROPERABILITY OF BROADBAND SATELLITE COMMUNICATION NETWORKS
}

\author{
William D. Ivancic, Daniel R. Glover, Thomas M. VonDeak, Kul B. Bhasin \\ NASA Lewis Research Center \\ Cleveland, Ohio 44145
}

\begin{abstract}
The realization of the full potential of the National Information Infrastructure (NII) and Global Information Infrastructure (GII) requires seamless interoperability of emerging satellite networks with terrestrial networks. This requires a cooperative effort between industry, academia and government agencies to develop and advocate new, satellite-friendly communication protocols and modifications to existing communication protocol standards. These groups have recently come together to actively participating in a number of standards making bodies including: the Internet Engineering Task Force (IETF), the Asynchronous Transfer Mode (ATM) Forum, the International Telecommunication Union (ITU) and the Telecommunication Industry Association (TIA) to ensure that issues regarding efficient use of these protocols over satellite links are not overlooked. This paper will summarize the progress made toward standards development to achieve seamless integration and accelerate the deployment of multimedia applications.
\end{abstract}

\section{Introduction}

The state-of-the-art in satellite communications offer reliable telephony, video and data transmissions. Single application is provided in the link, what is known as a vertical layer network architecture. However, the emerging broadband or multimedia applications over proposed satellite networks require horizontal layer network architecture (as shown in Figure 1) in order for the network users and providers to set up reliable connections for desired applications.

\begin{tabular}{|c|}
\hline Application \\
\hline Presentation \\
\hline Session \\
\hline Transport \\
\hline Network \\
\hline Data Link \\
\hline Physical \\
\hline
\end{tabular}

Figure 1

Current communication protocols such as ATM and the Transmission Control Protocol / Internet Protocol (TCP/IP) protocol suite are currently being utilized to achieve horizontal network architecture over satellite links. However, there is ample room for improvement in the efficiency 'of these protocols over satellite links. Modifications are necessary to improve protocol performance over long delay, non-error-free links. Industry, academia and government agencies (referred to in this paper as the Coalition) are performing research to understand the interaction of various protocols relative to satellite environments and recommend improvements at the appropriate protocol layers. The goals are: 1) to develop modifications to current protocols and develop new protocols that benefit the entire telecommunication industry including the satellite industry; and 2) to have these protocols implemented directly into the general users' computers and communication systems. In order to reach these goals, the new protocols and protocol modifications must become universally accepted by industry. This is only possible by working closely with computer and telecommunications industry and the standards making bodies such as the Internet Engineering Task Force, the ATM Forum, the Telecommunication Industry Association and other standards making bodies.

\section{Architectural Framework for Seamless Interoperable Satellite Networks} The methodology of addressing the large scope of interoperability for global broadband satellite network is to develop architectural framework from which the key interoperability issues at each of the layers and respective interfaces can be identified. Advanced satellite network architectures are under development in various NII/GII forums. These advance architecture concepts will be utilized by industry and government agencies to develop standards and to direct technology developments into the $21^{\text {st }}$ century.

Contributions on top-level architectural framework have also been made to the American National Standards Institute (ANSI) Information Infrastructure Standard Panel (IISP) for identification of specifications and standards development organizations and to the ITU identifying satellite scenarios relative to the national and global information infrastructure

Efficiency is defined as the ability of fully utilize the available transmission bandwidth. 
(NII/GII). ${ }^{1,2}$ Such scenarios are being used in defining standard needs and gaps. An example of satellite scenarios are given below.

Asynchronous Transfer Mode (ATM) Standards ATM is a protocol originally conceived by the telecommunication industry to handle multimedia traffic over wide area networks (WANs). The protocol is a connection oriented cell switched ${ }^{\dagger}$ protocol developed for fiber optic systems which have near-error-free performance characteristics. The protocol encompasses features of both the data link layer and the routing layer, layers 2 and 3 of the Open Systems Interface (OSI) model.

The main features of ATM are guaranteed quality of service (QoS), ease of switching and multimedia compliance. The ATM cell structure of 53 bytes -- a 5 byte header and 48 byte payload -- was designed for high bandwidth implementations with low switching and routing overhead. The 5 byte header of each cell contains all the information necessary for the end-to-end delivery of the cell. If the header of the cell is corrupted beyond the corrective abilities of the included 1 byte CRC check then the cell is discarded. The small header and limited routing rules allow the switching to be performed in high speed hardware. The small size of the ATM cell -- 53 bytes -- allows small internal buffers to be used in the switches keeping cell delay and jitter to a minimum. ATM requires the order of ATM cells must be maintained and that the link be nearly errorfree. These requirements directly affect the design of satellite and wireless networks that wish to utilize ATM.

The following interrelated issues must be addressed for ATM to be utilized over satellite (and other wireless) links:

- Reference Models

- Quality of Service

- Traffic Management and Congestion Control

- Routing

- Handover

- Signaling

- Multicasting

- Security

The Coalition is developing reference models and performing research relative to ATM Quality of Service (QoS), signaling, routing, congestion management, and architecture issues relative to ATM and reporting the results to both the ATM Forum and ITU.

${ }^{\dagger}$ Cell Switching is defined as a subset of packet switching where all packets are of one fixed size.
Reference Models

The Coalition has been working to develop various reference models that identify common interface points (common air, protocol layer, and signaling) necessary for hardware and protocol development. The reference models provide a common basis for discussion and represent the industry's current thinking on the development of satellite based ATM architectures. Figure 1 is one example of the type of reference models being defined -- this model depicts the components and interfaces involved in supporting a ground based mobile ATM switch access into the fixed network through a satellite. Most of the recent work has been in support of the Telecommunication Industry Association's Communications Interoperability Subcommittee of the Satellite Communication Division (TIA/SCD/CIS) and has been related to ATM networks. ${ }^{3}$ These contributions have also been submitted to the ITU-R Working Party $4 \mathrm{~B}$ and are being incorporated into the ITU-R Draft Recommendation S.atm.

The Coalition, through the TIA/SCD/CIS ATM working group, is defining satellite ATM networks requirements and architectures. The output of this work is being utilized by the ATM Forum to address protocol

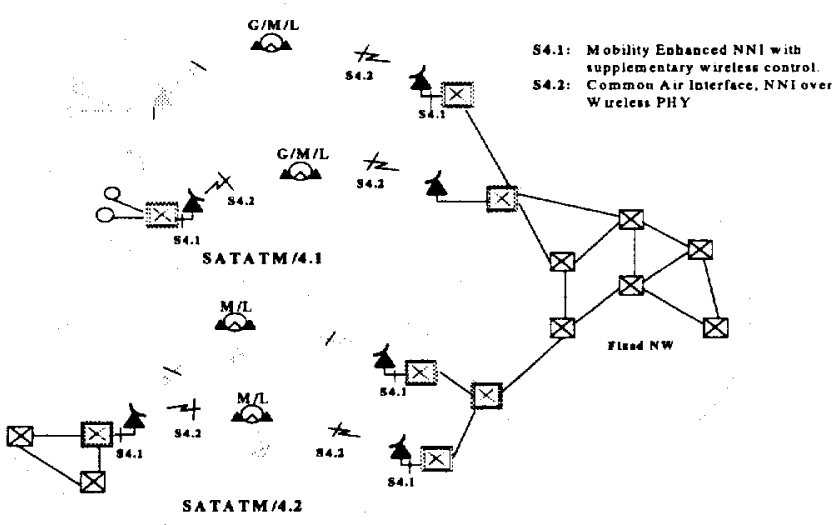

Figure 1 - ATM Common Air Interface Reference Model

modifications for signaling and network management and will eventually feed into the ITU standards. An additional contribution has been made to the ATM Forum in response to questions from some members of the Wireless ATM (WATM) Working Group regarding the spectrum that would be used by the satellite industry providing ATM based services. ${ }^{4}$ This particular contribution indicates that satellites have been successfully utilized to transmit data using ATM protocols and that systems currently exist that can be used for such applications that require ATM. This contribution also provides a list of future systems that 
plan to carry data over satellites at rates up to OC-48 (2.4 Gbps).

In the future, the Coalition plans to continue supporting the TIA and the ATM Forum regarding reference model definition and further develop many of the details of currently accepted models.

\section{Quality of Service (QoS)}

NASA Lewis Research Center has recently performed experiments using MPEG-2 compressed digital video over ATM to determine the link quality that satellites must provide for Class 1 "stringent class" services over ATM $^{5}$ [Figure 2]. The QoS issues are being directly reported to the industry and US contingent of the ITU-R working party $4 \mathrm{~B}$.

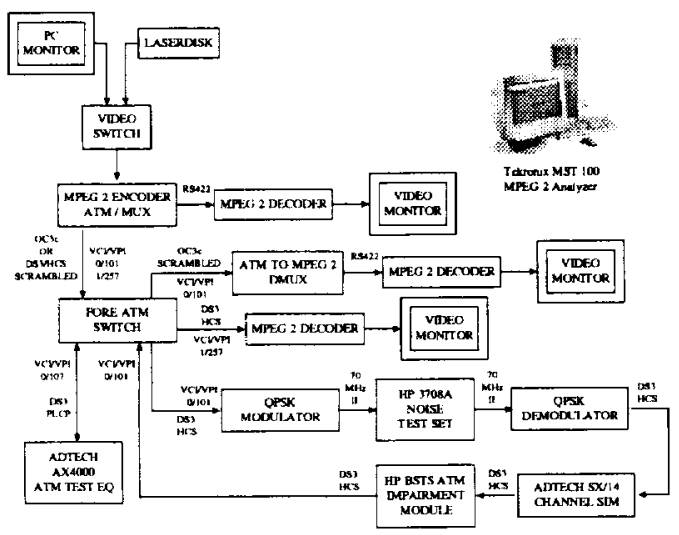

Figure 2 - ATM QoS Testing Using MPEG-2

Recent contributions to the ITU include work related to QoS and routing as well as to aforementioned reference configuration model for wireless ATM work. ${ }^{6}$ These contributions are being integrated into the ITU-R Recommendation S.atm dealing with B-ISDN ATM performance requirements and S.atm-av dealing with $B$ ISDN ATM availability requirements. By request of the ITU-R 4B chairperson, the QoS contributions have also been submitted to the T1A1.3 United States standards group that works on ATM performance issues and is responsible for United States contributions to the ITU-T on ITU-T Recommendation 1.356, "B-ISDN ATM Layer Cell Transfer Performance."

\section{Traffic Management}

Recent NASA sponsored contributions to the ATM Forum include reroute and traffic management mechanisms related to ATM over satellites. ${ }^{7,8}$ and 9 Much of this work resulted from an Ohio State University / NASA partnership with Ohio State University addressing traffic management and congestion control protocol issues relative to ATM over satellite. ${ }^{10}$ Ohio State University is also working closely with the ATM switch manufacturers to ensure that new algorithms and techniques are implemented in commercial hardware.

\section{Future Research}

Future Coalition research will be directed toward ATM QoS requirements for TCP/IP and other internet protocols as well as signaling, routing, handovers and traffic management for various satellite network configurations such as bandwidth on demand, MFTDMA access, scanning beams and Low Earth Orbit (LEO) and Medium Earth Orbit (MEO) networks.

Data Protocol Standards

The Coalition's objective regarding data protocol standards is to allow multimedia-based applications to seamlessly operate over satellites with terrestrial networks by overcoming limits in widely used protocols caused by satellite characteristics (delay, noise, etc.). Efficiency issues relative to Internet protocols such as TCP and HTTP (HyperText Transfer Protocol) are currently being addressed. "

\section{Transmission Control Protocol /nternet Protocol (TCP/IP)}

TCP/IP is the primary protocol suite used today over the Internet. TCP is a reliable protocol ${ }^{\ddagger}$ designed to work in a variety of environments; thus, TCP has always worked in satellite networks. However, since all reliable protocols require some type of feedback to acknowledge successful data reception, most general implementations of $T C P^{\delta}$ perform inefficiently in networks that have large delays relative to the available transmission bandwidth (known as the bandwidth-delay product). A high bandwidth-delay product connection can result from a very high data rate just as well as from a high delay. A network having a delay-bandwidth product that significantly exceeds $10^{-5}$ bits is consider a long fat network (LFN) and requires known and accepted extensions to general implementations of TCP in order to operate efficiently.

Many tuning parameters are available to enhance the performance of TCP, including segment size, timers and window sizes. There are also numerous congestion avoidance algorithms such as slow start, selective retransmission and selective acknowledgment that can

\footnotetext{
$\ddagger$ A reliable protocol is one that guarantees accurate delivery of information.

${ }^{\delta}$ A general implementation of TCP does not have the necessary extensions to allow for efficient transmission over network that have a large bandwidth-delay product.
} 
be utilized to improve performance. To date these tuning parameters and control algorithms have been optimized for error-free networks -- usually for local area networks.

The Coalition is investigating the performance of various transmission and congestion algorithms over long delay, error-prone and near-error-free satellite networks in order to advocate proposed modifications to the TCP protocol suite as well as suggesting new modifications and recommending settings for the tuning parameters. To support this effort, the NASA Lewis Research Center and Ohio University are working together to evaluate Internet Protocols over satellite networks utilizing NASA's Advance Communication Technology Satellite. Figure 3 shows a detailed layout of our one of the Internet Protocol test network utilized by Ohio University and Lewis Research Center. In addition, BBN is performing research to improve TCP over long latency links and is active in the IETF advocating improvements to the Internet protocols that are favorable to the satellite community.

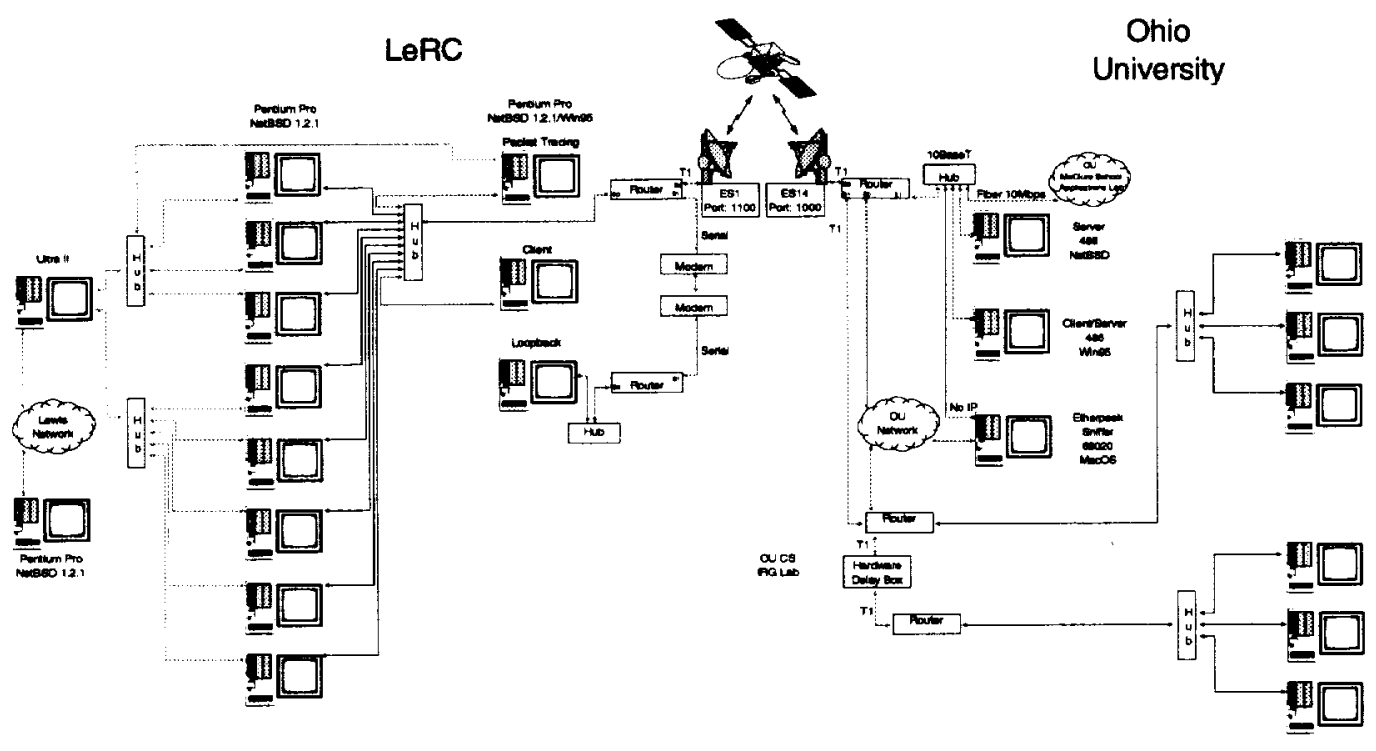

Figure 3 - NASA Lewis Internet Protocol Test Network (Low Rate Portion)

The Coalition was instrumental in helping establish the IETF's TCP Over Satellite Working Group (TCPSAT). TCPSAT is working on a best common practices document describing TCP over satellite and listing research topics. "The objective of this group is to decide how to best address known problems in existing implementations of the current TCP standard(s) and practices. The overall goal is to improve conditions in the existing Internet by enhancing the quality of current TCP/IP implementations. It is hoped that both performance and correctness issues can be resolved by making implementers aware of the problems and their solutions. In the long term, it is felt that this will provide a reduction in unnecessary traffic on the network, the rate of connection failures due to protocol errors, and load on network servers due to time spent processing both unsuccessful connections and retransmitted data. This will help to ensure the stability of the global Internet."

Recent contributions to TCPSAT have been directed at enhancing TCP over satellite channels using standard mechanisms. ${ }^{12}$ and 13 At the IETF meeting of August 11, 1997, in Munich, Germany, a proposal was given by Von Jacobson suggesting an ACK spacing algorithm that could be used at a satellite ground station on the downlink return path to help avoid burstiness that can occur when using large

\footnotetext{
" http://www.ietf.org/html.charters/tcpimpl-
} charter.html, Last Modified: 29-Jul-97 
windows. This is an extremely important issue to satellite and requires additional research as to the proper algorithms and trigger points for implementation.

The TCP Implementation Group (TCPImpl) is also working to identify non-standard TCP as well as performing research on proposed TCP modifications. An example of this is the NASA Lewis Research Center's research on a draft to increase the initial window size that should help speed up TCP's slow start performance.

\section{Future Plans}

Future plans include evaluating and experimenting with TCP at higher rates and evaluating popular application protocols such as HTTP1.1. Another area of research that will be addressed is reliable multicast over satellite. This is of major importance to the satellite industry as satellites provide an ideal transport mechanism for broadcast and multicast services.

\section{Summary}

A brief synopsis of the current progress toward standards has been given along with the references for details on any particular subject. Additional information and many of these references are available via the World Wide Web from the Satellite Networks and Architectures Branch of NASA Lewis Research Center's Communication Technology Division. ${ }^{14}$ Future protocol work will be directed at satellite network access and interoperability with terrestrial systems as well as multicast protocols. Both these areas are necessary to enable existing and new communication services via satellite at a competitive price.

\section{References}

1. R. Jirberg, K. Bhasin and E. Bobinsky: "Tool Kit for Identification of Specification and Standards Development Organizations for the NII/GII," January 1996.

2. Bhasin and E. Bobinsky: "Additional Satellite Scenarios for I.GII," Scen. Meth Document of ITU's Joint Rapporteurs' Group on GII submitted and accepted.
3. M. Barton, D. Pinto, D. Kostas, T. VonDeak and S. Hulyalkar: "Reference Configuration Model for Wireless ATM," ATM Forum Doc. No. 961623, Melbourne.

4. Lowry and T. VonDeak: "Satellite ATM Utilization,” ATM Forum Doc. 97-0358.

5. ITU-T I.356: B-ISDN ATM Layer Cell Transfer Performance.

6. Ivancic, B. Frantz and M. Spells: "MPEG-2 Over ATM Over Satellite QoS Experiments: Laboratory Tests," United States of America Contribution to International Telecommunication Union Radio Frequency (ITU-R) Working Party 4B, Philadelphia, PA., October 1997, T1A1 Contribution T1A1.3/97-084, October 1997.

7. T. vonDeak: "Generic Reroute Mechanism," Doc. ATM Forum No. 97-0651.

8. G. Babic, A. Durresi, R. Jain, J. Dolske and S. Shahpurwala: "ATM Switch Performance Testing Experiences," ATM Forum Doc. No. 970178R1.

9. S. Kalyanaraman, R. Jain, R. Goyal, S. Fahmy and S. Kim: "Virtual Source/Virtual Destination (VS/VD): Design Considerations," ATM Forum Doc. No. 96-1759.

10. http://www.cis.ohio-state.edu/ -jain/papers, October 1997.

11. Bhasin, D. Glover and E. Bobinsky: "The Internet and Next-Generation Satellite Systems," Proceedings of the $12^{\text {th }}$ Annual IEEE Workshop on Computer Communications, pp. 1-9, September 1997.

12. Glover and M. Allman: "Enhancing TCP Over Satellite Channels Using Standard Mechanisms," Internet Draft, Work-in-progress.

13. Allman, R Durst and E. Travis: "Issues Related to TCP Performance in a Satellite Environment and Discussion of Possible Protocol-Oriented Mitigations," Presentation at $39^{\text {th }}$ IETF Meeting, Munich, Germany, August 1997.

14. http://sulu.lerc.nasa.gov/5610/5610.html, October 1997. 


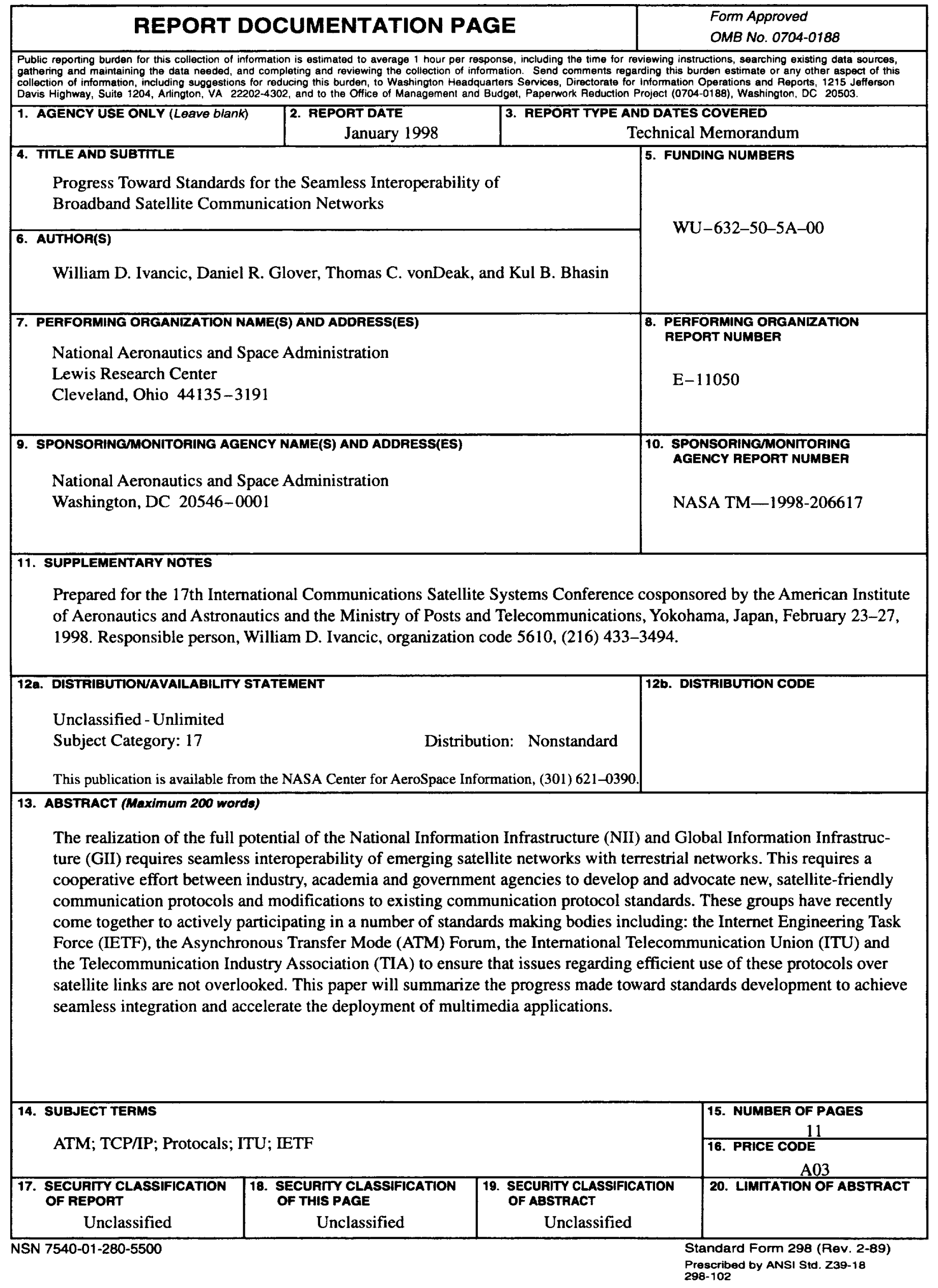

